\section{Nuclear Gene Indicates Coat-Color Polymorphism in Mammoths} Holger Römpler, ${ }_{1}^{1}$ Nadin Rohland, ${ }^{2}$ Carles Lalueza-Fox, ${ }^{3}$ Eske Willerslev, ${ }^{4}$ Tatyana Kuznetsova, $^{5}$
Gernot Rabeder, ${ }^{6}$ ]aume Bertranpetit, ${ }^{7}$ Torsten Schöneberg, ${ }^{1}$ Michael Hofreiter ${ }^{2 *}$

$\mathrm{B}$ ecause more than $99 \%$ of all species that have ever lived on Earth are extinct, the genetic basis of most phenotypic traits that have evolved during life's history cannot be studied directly. Although it is possible to approximately reconstruct the gene sequences of extant species' common ancestors (1), many extinct species represent end points of evolution, and their gene sequences cannot be investigated by using this approach. For late Pleistocene species, ancient DNA techniques should in principle allow analyses of extinct genes. However, despite progress in the analysis of ancient DNA, to date only short fragments of nuclear genes could be amplified from Pleistocene specimens $(2,3)$.

We have combined two methods recently introduced in ancient DNA research, large-scale extraction and multiplex polymerase chain reaction (PCR), to amplify 1236 base pairs (bp), including the complete coding sequence, of the single-exon nuclear melanocortin type 1 receptor $(\mathrm{Mcl} / \mathrm{r})$ gene from an 43,000-year-old mammoth (Mammuthus primigenius) bone from Siberia. The product of the Mclr gene is a key protein in the determination of hair color in both humans and other animals (4), providing an opportunity to connect genotype and phenotype in extinct taxa. For example, reduced activity of MC1R has been shown to result in red hair in humans and cows and red or yellow hair in mice, horses, and dogs (4). Dark- and light-colored mammoth hair has been recovered from permafrost mummies, giving circumstantial evidence that mutations may occur in one of the genes involved in determination of hair color. We amplified the mammoth Mclr gene in 23 primary fragments varying in length from 101 to $138 \mathrm{bp}$. Three positions were found to be polymorphic in the individual sequenced, all resulting in amino acid changes $\left[\mathrm{Thr}^{21} \rightarrow \mathrm{Ala}^{21}\right.$ (Thr $\left.{ }^{21} \mathrm{Ala}\right), \mathrm{Arg}^{67} \mathrm{Cys}$, and
$\mathrm{Arg}^{301} \mathrm{Ser}$, positions relative to the elephant $\mathrm{Mclr}$ sequence] (Fig. 1A) (5). Because template damage may affect ancient DNA sequences (6), we sequenced each polymorphic position from at least three independent PCRs and, by using singlenucleotide polymorphism (SNP) typing, from additional specimens. The identification of homozygous individuals from these additional mammoth samples revealed most likely allelic constitutions of $\mathrm{Thr}^{21}-\mathrm{Arg}^{67}-\mathrm{Arg}^{301}$ (allele 1) and $\mathrm{Ala}^{21}-\mathrm{Cys}^{67}$ $\mathrm{Ser}^{301}$ (allele 2), respectively (5). Whereas $\mathrm{Thr}^{21}$ is highly variable among mammalian MC1R orthologs, substitutions of $\mathrm{Arg}^{67}$ and $\mathrm{Arg}^{301}$ (Fig. 1A) affect highly conserved residues. To investigate whether the two alleles show a functional difference, we constructed Mclr expression vectors that are identical to the inferred mammoth alleles on the amino acid level. The product of allele 1 shows high basal activity and robust responses to two agonists, $\alpha \mathrm{MSH}$ (Fig. 1B) and NDP- $\alpha$ MSH (5), in a concentrationdependent manner. In contrast, the product of allele 2 shows an almost complete loss in basal activity and an $\sim 65 \%$ reduction in efficacy $\left(E_{\max }\right)$ to both agonists. Functional testing of each position variable
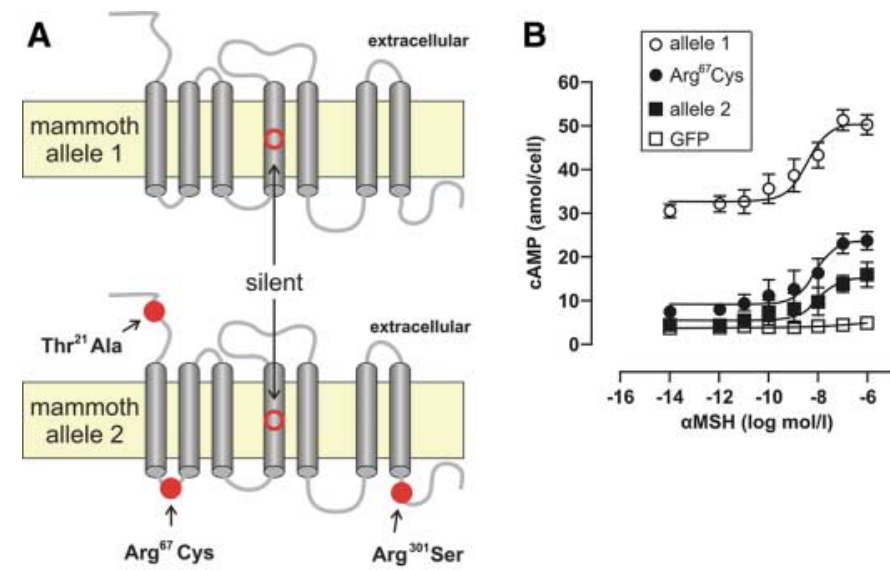

Fig. 1. (A) Localization of the fixed and polymorphic positions in mammoth Mc1r. The seven transmembrane domain architecture of MC1R is depicted. Compared with the Mc1r sequence of the African elephant, the approximate positions of the fixed silent position and polymorphic positions in the two mammoth alleles are circled. (B) Partial loss of function in mammoth MC1R variants. For functional characterization of the wild type (allele 1), $\mathrm{Arg}^{67}$ Cys and $\mathrm{Thr}^{21} \mathrm{Ala}-\mathrm{Arg}^{67} \mathrm{Cys}-\mathrm{Arg}^{301}$ Ser (allele 2) constructs of the Mc1r gene were transiently transfected into COS-7 cells and tested for agonist-induced cyclic adenosine monophosphate (cAMP) accumulation. Green fluorescent protein (GFP) plasmid-transfected cells served as controls. Intracellular CAMP was measured in response to increasing concentrations of $\alpha-M S H$ as described (5). Shown is the mean \pm SEM of four independent experiments, each performed in triplicate. in the mammoth individually and all possible combinations of the three mammoth-specific amino acids revealed the $\mathrm{Arg}^{67} \mathrm{Cys}$ substitution as the main cause for MC1R dysfunction (Fig. 1B) (5).

Pleistocene mammoths harbored both a fully and a partially active MC1R variant. Given an otherwise functional pathway for hair coloration, the reduction in activity of the $\operatorname{Arg}^{67} \mathrm{Cys}$ variant would be sufficient to result in substantially lighter hair color. Intriguingly, light-colored populations of the beach mouse (Peromyscus polionotus leucocephalus) carry an $\mathrm{Mclr}$ allele that contains an Arg-to-Cys substitution at the homologous sequence position, and functional tests and crossing experiments have revealed both a reduction in basal and induced activity highly similar to that observed for the mammoth MC1R protein and a strong association between this amino acid polymorphism and adaptive coat color phenotype (7). These findings strongly argue for a phenotypic effect of this mutation also in the mammoth. Thus, it is possible that mammoth populations were polymorphic with regard to hair color, harboring both dark- and light-haired individuals. Although it is currently impossible to determine whether hair color was adaptive in mammoth, our study shows that functional analyses of nuclear genes are possible from late Pleistocene species. This opens up the possibility of studying a wide range of extinct species' features invisible in the fossil record.

\section{References and Notes}

1. J. W. Thornton, Nat. Rev. Genet. 5, 366 (2004).

2. A. Greenwood, C. Capelli, G. Possnert, S. Pääbo, Mol. Biol. Evol. 16, 1466 (1999)

3. H. N. Poinar et al., Science 311, 392 (2006)

4. J. L. Rees, Annu. Rev. Genet. 37, 67 (2003).

5. Materials and methods are available as supporting material on Science Online.

6. M. Hofreiter et al., Nucleic Acids Res. 29, 4793 (2001).

7. H. E. Hoekstra, R. J. Hirschmann, R. A. Bundey, P. A. Insel, J. P. Crossland, Science 313, 101 (2006).

8. We thank the members of our research groups and several anonymous reviewers for comments that improved the manuscript. This work was funded by the Max Planck Gesellschaft, Bundesministerium für Bildung, Wissenschaft, Forschung und Technologie and Deutsche

Forschungsgemeinschaft. Sequences have been deposited in GenBank under accession nos. DQ648859 to DQ648866.

\section{Supporting Online Material}

www.sciencemag.org/cgi/content/full/313/5783/62/DC1

Materials and Methods

Tables S1 and S2

References

20 April 2006; accepted 30 May 2006

10.1126/science. 1128994

${ }^{1}$ Molecular Biochemistry, Institute of Biochemistry, Medical Faculty, University of Leipzig, 04103 Leipzig, Germany. 'Department of Evolutionary Genetics, Max Planck Institute for Evolutionary Anthropology, 04103 Leipzig, Germany. ${ }^{3}$ Departament de Biologia Animal, Facultat de Biologia, Universitat de Barcelona, 08028 Barcelona, Spain. ${ }^{4}$ Ancient DNA and Evolution Group, Centre for Ancient Genetics, Niels Bohr Institute and Biological Institute, University of Copenhagen, 2100 Copenhagen, Denmark. ${ }^{5}$ Department of Paleontology, Faculty of Geology, Moscow State University, 119992 Moscow, Russia. 'Department of Paleontology, University of Vienna, 1090 Vienna, Austria. ${ }^{7}$ Unitat de Biologia Evolutiva, Facultat de Ciències de la Salut i de la Vida, Universitat Pompeu Fabra, 08003 Barcelona, Spain.

*To whom correspondence should be addressed. E-mail: hofreite@eva.mpg.de 